\section{Autoavaliação positiva de estresse e prática de atividades físicas no lazer em estudantes universitários brasileiros}

\section{Positive self-rated stress and leisure-time physicals activities in undergraduate from Brazil}

\author{
Glauciambérguia de Almeida Pirajá1, \\ Thiago Ferreira de Sousa ${ }^{2,3}$ \\ Silvio Aparecido Fonseca ${ }^{2,3}$ \\ Aline Rodrigues Barbosa ${ }^{2}$ \\ Markus Vinicius Nahas ${ }^{2}$
}

\section{Resumo}

Os objetivos desta pesquisa foram: i) estimar a prevalência de autopercepção positiva de estresse; ii) analisar a associação entre os fatores sociodemográficos e de vínculo com a universidade com a autopercepção positiva do estresse; e, iii) analisar a associação entre a prática de atividade física no lazer em diferentes intensidades com a autopercepção positiva de estresse. Este estudo de delineamento transversal foi realizado em 2010 com uma amostra de graduandos de uma instituição pública de ensino superior do Estado da Bahia. A amostra calculada foi de 1.232 estudantes universitários e a amostragem foi estratificada e proporcional aos cursos, período de estudo e ano de ingresso na universidade, com posterior seleção dos sujeitos por seleção aleatória simples em cada estrato. $\mathrm{O}$ desfecho deste estudo (autoavaliação de estresse positivo na vida) foi analisado em relação aos indicadores sóciodemográficos, de vínculo com a universidade e com a prática de atividades físicas no lazer. A Razão de Prevalência foi a medida de associação empregada. Dentre os 1.084 graduandos participantes do estudo verificou-se maiores proporções de autopercepção de estresse positivo em homens, estudantes de 17 a 20 anos e que entraram na universidade no ano de 2010. Os estudantes que praticaram atividades físicas em intensidade vigorosa e, moderada e vigorosa apresentaram maiores prevalências de autopercepção de estresse positivo que os estudantes inativos no lazer. Conclui-se que a prática de atividades físicas no lazer moderadas a vigorosas associou-se com o estresse positivo percebido, assim como os estudantes do sexo masculino, jovens e recém-ingressantes na universidade.

\section{Palavras-chave}

Estresse da vida, Atividade motora, Estudantes, Estudos transversais.

\begin{abstract}
The objectives were: i) to estimate the prevalence of positive self-rated stress, ii) to analyze the association between sociodemographic factors and program affliation of the university with a positive self-rated of stress, and, iii) to analyze the association between the leisure-in-time physical activity practice at different intensities with positive self-rated of stress. This cross-sectional study was conducted in 2010 with a sample of students at a public university from State of Babia, Brazil. The sample was of 1,232 college students and the sample was stratified and proportional to the courses, study period and year of entry into the university and the students selected in each stratum by simple random selection. The outcome of this study was the self-rated of positive stress in life and analyzed in relation to sociodemographic, program affiliation with the university and leisure-in-time physicals activities. The prevalence ratio was the measure of association employed. Participated 1,084 students and higher proportions of positive self-perception of stress were observed in men, students 17-20 years and who entered the university in 2010. Students who practiced in vigorous, and moderate and vigorous physicals activities had higher prevalence of positive self-rated stress those students inactive in leisure. It is concluded that leisure-in-time physicals activities practice in moderate to vigorous intensities were strongly associated with positive stress, as well as the male students, young and newly entering the university.
\end{abstract}

\section{Keywords}

Stress, Psychological, Physical activity, Students, Cross-sectional studies.
Rev Bras Ativ Fis Saúde p. 740-749 DOI:

http://dx.doi.org/10.12820/rbafs.v.18n6p740

1Pós-Graduação em Metodologia em Educação Física e Esporte da Universidade Estadual de Santa Cruz, Ilhéus, Bahia.

2Programa de Pós-Graduação em Educação Física da Universidade Federal de Santa Catarina, Florianópolis, Santa Catarina. 3Universidade Estadual de Santa Cruz, Ilhéus, Bahia. 


\section{INTRODUÇÃO}

No ano de 1936 o fisiologista Hans Selye tornou conhecido o termo "stress" (ou estresse) palavra originada do latim stringere ${ }^{1,2}$. O estresse é a maneira como o organismo responde a qualquer estímulo - bom, ruim, real ou imaginário - que altere seu estado de equilibrio ${ }^{2}$. $\mathrm{O}$ estresse pode apresentar sensações físicas que quando combinadas com fatores emocionais e psicossociais resultam em estímulos de medo, pressões psicológicas, preocupações, ansiedades, fadigas físicas sem motivo aparente, que de certo modo vão exigir do corpo e/ou organismo uma adaptação ${ }^{1}$. Para que esta adaptação ocorra é necessário forte estímulo de energia física, mental e social ${ }^{3}$.

Existem incontáveis situações e experiências que podem dar origem ao estresse, diferenciado apenas pelo modo que cada indivíduo reage aos fatores estressores ${ }^{4}$. O estresse, no conceito moderno, se manifesta de acordo com as características individuais e interação com o ambiente social, ou seja, um processo biológico, psicológico e social ${ }^{2}$. Por ser um aspecto natural da própria vida, o estresse não é algo que deva ser ignorado, nem tampouco evitado. Embora possa ser uma ameaça à saúde, também pode ser um estímulo necessário às adaptações positivas no organismo ${ }^{2}$. O estresse pode ser subdividido em estresse positivo (eustress) e negativo $(\text { distress })^{2}$. O distress é o que acarreta ao corpo prejuízos orgânicos e assim a autopercepção positiva do estresse torna-se algo inconstante, e os efeitos destes sobre aspectos psicofisiológicos podem acarretar na diminuição da qualidade de vida e o aparecimento de doenças ${ }^{5}$, em contrapartida, o eutress pode ser mais comum quando ocorre à prática de exercícios físicos moderados ${ }^{2}$.

Dentre os grupos populacionais, os estudantes universitários apresentam maior suscetibilidade a condutas negativas à saúde, como baixos níveis de atividades físicas ${ }^{6,7}$. Além disso, estão sujeitos a diversas situações estressantes seja pela competividade, número de trabalhos acadêmicos, provas ou expectativa do mercado de trabalho ${ }^{8}$. Dentre os benefícios da prática de atividades físicas podem ser citados o controle das emoções, a execução de tarefas, melhora a função cognitiva e qualidade do sono ${ }^{9}$, elementos este que combinados auxiliam na autopercepção positiva de estresse, como observado em relação à percepção positiva de qualidade de vida ${ }^{10}$.

Considerando que os estudantes universitários podem estar mais sujeitos a situações estressantes e a carência de estudos sobre a associação entre a prática de atividades físicas no lazer e estresse em universitários brasileiros, a hipótese do presente estudo é que estudantes universitários ativos no lazer estão suscetíveis a maiores níveis de autoavaliação de estresse positivo na vida. Os objetivos desta pesquisa realizada com estudantes universitários de uma instituição de ensino pública do Estado da Bahia foram: i) estimar a prevalência de autoavaliação positiva de estresse; ii) analisar a associação entre os fatores sociodemográficos e de vínculo com a universidade com a autoavaliação positiva de estresse; e, iii) analisar a associação entre a prática de atividades físicas no lazer em diferentes intensidades com a autoavaliação positiva de estresse.

\section{MÉTODOS}

Este estudo transversal é resultado do inquérito realizado em 2010 da pesquisa longitudinal Monitoramento dos Indicadores de Saúde e Qualidade de Vida em Acadêmicos (MONISA), realizada em uma instituição de ensino superior públi- 
ca, localizada no Estado da Bahia. Maiores detalhes sobre os métodos do estudo foram descritos por Sousa et al. ${ }^{11}$. O Estudo MONISA foi aprovado pelo Comitê de Ética em Pesquisa local sob o número de protocolo 382/10.

A população do estudo foram os estudantes universitários matriculados nos cursos presenciais de graduação, no segundo semestre letivo do ano de 2010. Não fizeram parte da pesquisa os estudantes universitários ingressantes na instituição no segundo semestre do ano (calouros), os estudantes dos cursos de ensino a distância (EAD) e os alunos com matrícula especial.

Para o cálculo amostral ${ }^{12}$ foi considerada a população de referência $(\mathrm{N}=5.461)$, nível de confiança em 95\%, prevalência geral de 50\%, tendo em vista o emprego de diferentes desfechos relacionados à saúde e erro amostral de três pontos percentuais. A amostra obtida foi de 893 indivíduos, sendo incluídos 20\% para possíveis perdas e recusas e, $15 \%$ para o controle de fatores de confusão nas análises ajustadas. A amostra final necessária foi de 1.232 estudantes. Considerando a quantidade de estudantes nas categorias da variável atividade física no lazer, poder de $80 \%$ e nível de confiança de $95 \%$, a amostra deste estudo pode estimar Razões de Prevalências significativas, nas análises brutas, acima de 1,7. O cálculo do poder da amostra foi realizado a posteriori no programa Epi Info 7.

O processo de amostragem foi estratificado e proporcional aos 30 cursos presenciais da instituição, período de estudo (diurno e noturno) e, ano de ingresso na instituição (distribuídos em 2010, 2009, 2008 e, 2007 e anterior). Na população-alvo, para cada curso, de acordo com o período de estudo, uma quantidade de estudantes universitários representava proporcionalmente cada categoria referente ao ano de ingresso na universidade (por exemplo, estavam matriculados no curso de Licenciatura em Educação Física, do período de estudo diurno e ano de ingresso 2009, 38 estudantes universitários). Considerando a distribuição proporcional da população-alvo foi estimada a amostra em cada categoria de ano de ingresso, para cada um dos cursos de acordo com período de estudo diurno e noturno, respeitando essa proporcionalidade. Conforme apresentado no exemplo anterior, para representar o curso de Licenciatura em Educação Física, do período de estudo diurno e ano de ingresso 2009, nove estudantes universitários eram necessários.

Os estudantes foram escolhidos de forma aleatória em cada estrato, sendo usada a lista de matrícula em ordem alfabética. A seleção dos estudantes foi realizada com o auxílio do programa Research Randomizer. Todos os selecionados foram procurados até três vezes, em dias e horários diferentes. Os estudantes não encontrados ou que não tiveram interesse em participar foram considerados como perdas e recusas e, não houve reposição para essas situações.

O treinamento com a equipe de coleta dados, composta por profissionais de Educação Física e discentes de diferentes cursos da instituição foi realizado nos meses de julho e agosto (2010). As coletas de dados (setembro a novembro de 2010) foram realizadas nas salas de aula da instituição, antes, durante, ou ao final das aulas. Quando o número de estudantes selecionados nas disciplinas era maior ou igual a 10 foi solicitada a permissão aos professores para a aplicação do instrumento. Nas outras situações o contato foi realizado diretamente com os estudantes selecionados. Em ambas as situações de aplicação, coletiva e individual, as condições locais para o autopreenchimento foram semelhantes e os estudantes foram assistidos pelos aplicadores e, sanadas as possíveis dúvidas. Possíveis vieses de aplicação nessas situações não foram observados. As informações foram obtidas por meio do questionário Isaq-A (Indicadores de Saúde e Qualidade de Vida de Acadêmicos ${ }^{13}$. O índice médio de validade desse questionário foi de $92 \%$, clareza 
96\% e, especificamente, para a variável dependente deste estudo o nível de reprodutibilidade observado foi de Kappa (k) de 0,55.

\section{Variável dependente}

A variável dependente deste estudo foi a autoavaliação de estresse na vida, mensurada pela pergunta "como você classifica o nível de estresse na sua vida?", sendo o desfecho a avaliação de estresse positivo (nunca estressado e raramente estressado).

\section{Variáveis independentes}

Sexo, faixa etária em terços (17 a 20 anos, 21 a 23 anos e 24 a 52 anos) e situação conjugal (sem parceiro e com parceiro). A renda pessoal dos estudantes em reais, de acordo com o salário mínimo em 2010 ( $<\mathrm{R} \$ 510$,00 e $\geq \mathrm{R} \$ 510,00)$; período de estudo (diurno e noturno); ano de ingresso na universidade (2010, 2009, 2008 e 2007 e anterior). A área de estudo, conforme classificação empregada pela instituição foi dicotomizada em Saúde e Outras (Exatas da Terra e Engenharias, Biológicas, Agrárias, Humanas, Sociais Aplicadas, Linguística, Letras e Artes), considerando a hipótese de que estudantes da área da Saúde apresentam melhores indicadores de saúde que seus pares ${ }^{14,15} \mathrm{e}$ a baixa frequência para algumas áreas de estudo.

A prática de atividades físicas no lazer foi verificada por meio de uma pergunta, com 17 opções de práticas e mais três opções abertas para a inclusão de outros tipos não contemplados na lista de opções de atividades. Posteriormente, as opções foram categorizadas quanto à intensidade das atividades físicas praticadas ${ }^{16}$, em pelo menos um dia de uma semana normal em: prática de pelo menos uma atividade física no lazer de intensidade moderada (atividade física no lazer moderada); prática de pelo menos uma atividade física de intensidade vigorosa (atividade física no lazer vigorosa); prática de uma atividade física no lazer de intensidade moderada e uma de intensidade vigorosa (atividade física no lazer moderada a vigorosa); e, não praticou, referente aos estudantes inativos no lazer. Os indicadores de reprodutibilidade dos tipos de atividades físicas praticadas no lazer foram: futebol, $\mathrm{k}=1,00$; voleibol, $\mathrm{k}=0,64$; basquetebol, $\mathrm{k}=0,65$; handebol, $\mathrm{k}=1,00$; ginástica em geral, $\mathrm{k}=0,53$; corrida em ambientes abertos, $\mathrm{k}=0,87$; ginástica aeróbica, $\mathrm{k}=0,47$; prática de bicicleta, $\mathrm{k}=0,35$; lutas ou artes marciais, $\mathrm{k}=0,84$; corrida em esteira, $\mathrm{k}=0,78$; surfe, $\mathrm{k}=1,00$; natação, $\mathrm{k}=0,65$; musculação, $\mathrm{k}=0,93$; e, caminhada em ambientes abertos, $\mathrm{k}=0,63$. A prática de tênis de quadra, caminhada em esteira e hidroginástica não foram observados indicadores de $\mathrm{k}$, pela ausência de caso em uma das categorias ${ }^{13}$.

A tabulação dos dados foi realizado no programa EpiData 3.1 e as análises dos dados executas no programa SPSS versão 16.0. As análises descritivas empregadas foram: frequências absolutas e relativas, médias, desvios padrão, e valores mínimos e máximos. $\mathrm{O}$ teste do qui-quadrado e qui-quadrado para tendência linear foram usados nas análises de associação entre as variáveis sociodemográficas e de vínculo com a universidade com a autoavaliação positiva de estresse. As Razões de Prevalências (análises bruta e ajustada) foram empregadas para estimar a associação entre a prática de atividades físicas no lazer e a autoavaliação positiva de estresse e na análise ajustada, as variáveis sociodemográficas e de vínculo com a universidade, que apresentaram valor de $\mathrm{p}<0,20$ no teste qui-quadrado e qui-quadrado para a tendência na associação com a autoavaliação positiva de estresse foram empregadas como variáveis de controle de forma simultânea. $\mathrm{O}$ valor de significância adotado foi de $\mathrm{p}<0,05$. 


\section{RESULTADOS}

Participaram do estudo 1.084 estudantes universitários com média etária de 22 anos $\pm 5,2$ anos (17 a 52 anos). A maioria dos estudantes eram mulheres (54,7\%), da faixa etária de 21 a 23 anos (37,4\%), sem companheiro $(86,4 \%)$, com renda mensal maior ou igual a um salário mínimo $(35,9 \%)$, com entrada na universidade em 2007 ou anterior (33,1\%), do período diurno $(67,8 \%)$ e de outras áreas de ensino não relacionadas à saúde $(88,4 \%)$ (Tabela 1$)$.

Tabela 1 - Características descritivas dos estudantes universitários. MONISA 2010.

\begin{tabular}{|c|c|c|}
\hline Variáveis & $n$ & $\%$ \\
\hline \multicolumn{3}{|l|}{ Sexo } \\
\hline Masculino & 491 & 45,3 \\
\hline Feminino & 592 & 54,7 \\
\hline \multicolumn{3}{|l|}{ Faixa etária (anos) } \\
\hline $17-20$ & 285 & 26,7 \\
\hline $21-23$ & 400 & 37,4 \\
\hline $24-52$ & 384 & 35,9 \\
\hline \multicolumn{3}{|l|}{ Situação conjugal } \\
\hline Com parceiro & 147 & 86,4 \\
\hline Sem parceiro & 937 & 13,6 \\
\hline \multicolumn{3}{|l|}{ Renda mensal (em reais) } \\
\hline$<510,00$ & 672 & 64,1 \\
\hline$\geq 510,00$ & 376 & 35,9 \\
\hline \multicolumn{3}{|c|}{ Ano de entrada na Universidade } \\
\hline 2010 & 233 & 21,5 \\
\hline 2009 & 267 & 24,6 \\
\hline 2008 & 225 & 20,8 \\
\hline 2007 e anterior & 359 & 33,1 \\
\hline \multicolumn{3}{|l|}{ Período de estudo } \\
\hline Diurno & 735 & 67,8 \\
\hline Noturno & 349 & 32,2 \\
\hline \multicolumn{3}{|l|}{ Área de estudo } \\
\hline Saúde & 126 & 11,6 \\
\hline Outras* & 958 & 88,4 \\
\hline
\end{tabular}

\%: Proporção; *Exatas da Terra e Engenharias, Biológicas, Agrárias, Humanas, Sociais Aplicadas, Linguística, Letras e Artes.

A prevalência de autopercepção de estresse positivo foi de $27,6 \%$. As proporções de autoavaliação de estresse positivo em relação à prática de atividades físicas no lazer são apresentadas na Figura 1. Aproximadamente 40\% dos estudantes que praticavam atividades físicas no lazer nas intensidades moderada e vigorosa apresentaram estresse positivo na vida.

A Tabela 2 apresenta a associação entre os indicadores sociodemográficos e de vínculo com a universidade com a autopercepção positiva de estresse. As maiores proporções de autopercepção de estresse positivo foram observados entre os homens $(35,8 \%)$, com idade entre $17-20$ anos (34\%) e que entraram na universidade no ano de 2010 (33\%). 
Tabela 2 - Análise de associação entre os indicadores sociodemográficos e de vínculo com a universidade com a autoavaliação de estresse positivo. MONISA, 2010.

\begin{tabular}{|c|c|c|c|}
\hline \multirow{2}{*}{ Variáveis } & \multicolumn{2}{|c|}{ Autoavaliação de estresse positivo } & \multirow{2}{*}{$\mathrm{p}$} \\
\hline & $\mathrm{n}$ & $(\%)$ & \\
\hline Sexo & & & $\leq 0,001^{\dagger}$ \\
\hline Masculino & 491 & $(35,8)$ & \\
\hline Feminino & 591 & $(20,8)$ & \\
\hline Faixa etária (anos) & & & $0,001^{*}$ \\
\hline $17-20$ & 285 & $(34,0)$ & \\
\hline $21-23$ & 399 & $(28,3)$ & \\
\hline $24-52$ & 384 & $(22,7)$ & \\
\hline Situação conjugal & & & $0,057^{+}$ \\
\hline Com parceiro & 147 & $(21,1)$ & \\
\hline Sem parceiro & 936 & $(28,6)$ & \\
\hline Renda mensal (em reais) & & & $0,057^{+}$ \\
\hline$<510,00$ & 672 & $(29,8)$ & \\
\hline$\geq 510,00$ & 375 & $(24,3)$ & \\
\hline Ano de entrada na universidade & & & $0,012^{*}$ \\
\hline 2010 & 233 & $(33,0)$ & \\
\hline 2009 & 267 & $(28,8)$ & \\
\hline 2008 & 225 & $(26,7)$ & \\
\hline 2007 e anterior & 358 & $(23,7)$ & \\
\hline Período de estudo & & & $0,844^{+}$ \\
\hline Diurno & 734 & $(27,8)$ & \\
\hline Noturno & 349 & $(27,2)$ & \\
\hline Área de estudo & & & $0,310^{+}$ \\
\hline Saúde & 126 & $(23,8)$ & \\
\hline Outras+ & 957 & $(28,1)$ & \\
\hline
\end{tabular}

\%: Prevalência; †Teste qui-quadrado para heterogeneidade; * Teste qui-quadrado para tendência; +Exatas da Terra e Engenharias, Biológicas, Agrárias, Humanas, Sociais Aplicadas, Linguística, Letras e Artes.

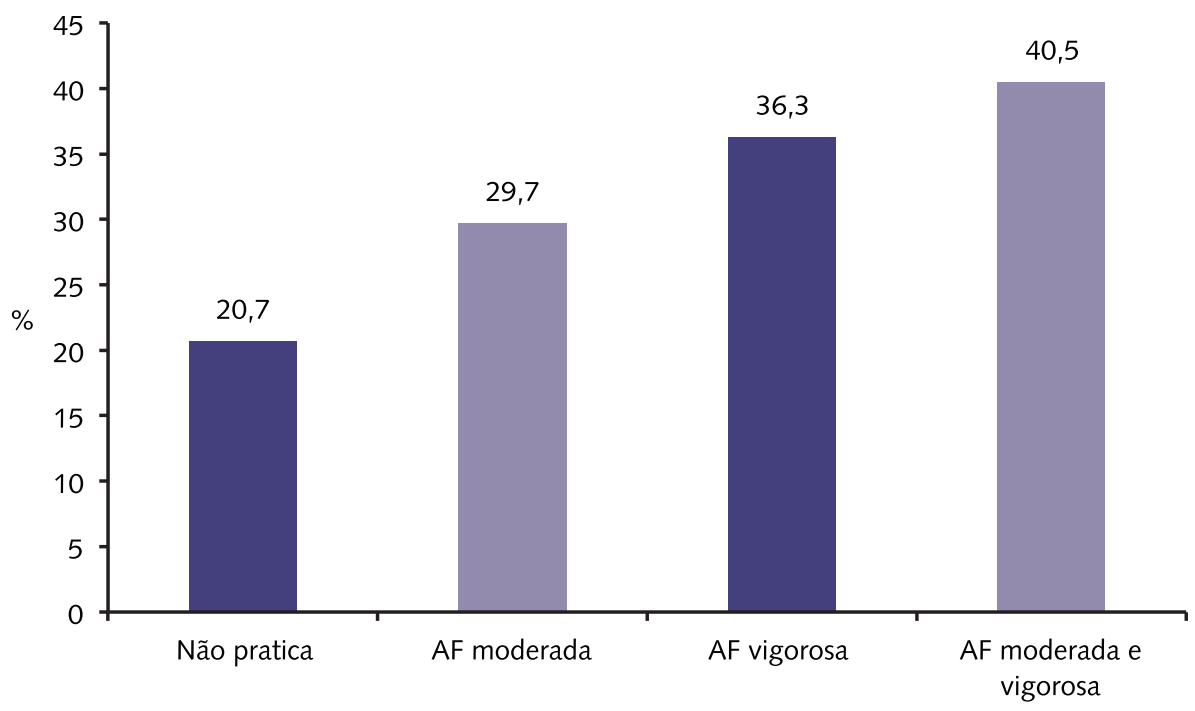

Figura 1 - Prevalência de autopercepção de estresse positivo de acordo a prática de atividades físicas no lazer. MONISA, 2010. AF: Atividade Física.

A Tabela 3 apresenta a associação entre a prática de atividade física no lazer e a autopercepção positiva de estresse. $\mathrm{Na}$ análise bruta, os estudantes que praticaram 
atividades físicas em intensidade vigorosa e moderada e vigorosa apresentaram maiores prevalências de autopercepção de estresse positivo que os estudantes inativos no lazer. $\mathrm{Na}$ análise ajustada (Tabela 3), a prevalência de autopercepção de estresse positivo foi $35 \%$ maior para os estudantes que praticaram atividade física vigorosa e $66 \%$ para os estudantes que praticaram atividade física moderada e vigorosa, quando comparados aos inativos no lazer.

Tabela 3 - Análise bruta e ajustada entre atividade física no lazer e autopercepção de estresse positivo em estudantes universitários. MONISA 2010. Razões de Prevalências estimadas por Regressão de Poisson.

\begin{tabular}{|c|c|c|c|c|c|}
\hline \multirow{3}{*}{ Variáveis } & \multicolumn{5}{|c|}{ Autopercepção de estresse positivo } \\
\hline & \multirow{2}{*}{$n$} & \multirow{2}{*}{$\frac{\text { Análise Bruta }}{\text { RP (IC95\%) }}$} & \multicolumn{3}{|c|}{ Análise Ajustada* } \\
\hline & & & $\mathrm{p}$ & RP (IC95\%) & $\mathrm{p}$ \\
\hline Atividade Física no lazer & & & $<0,001^{+}$ & & $0,002^{+}$ \\
\hline Não pratica & 540 & 1,00 & & $1,00^{\ddagger}$ & \\
\hline AF moderada & 209 & $1,43(1,10-1,87)$ & & $1,30(0,99-1,70)$ & \\
\hline AF vigorosa & 146 & $1,75(1,33-2,29)$ & & $1,35(1,01-1,80)$ & \\
\hline AF moderada e vigorosa & 163 & $1,95(1,52-2,50)$ & & $1,65(1,28-2,13)$ & \\
\hline
\end{tabular}

\%: Prevalência; RP: Razões de Prevalências; AF: Atividade Física; IC95\%: Intervalo de Confiança a $95 \%$; *Ajustada para as variáveis sociodemográficas e de vínculo com a universidade. ${ }^{\dagger} p$ valor do teste de Wald para heterogeneidade; ${ }^{\ddagger}$ Ajuste realizado para as variáveis que apresentaram valor de $p$ $<0,20$ do teste qui-quadrado e qui-quadrado para a tendência na associação apresentada na Tabela 2, sendo: sexo, faixa etária, situação conjugal, renda mensal em reais e ano de ingresso na universidade.

\section{DISCUSSÃO}

Os resultados mostraram que os homens, os mais jovens (17 a 20 anos de idade) e aqueles do início do curso apresentaram maiores prevalências de autopercepção de estresse positivo. Os estudantes que praticaram atividades físicas em intensidade vigorosa e, moderada e vigorosa apresentaram maiores prevalências de autoavaliação positiva de estresse que os estudantes inativos no lazer, independente do sexo, idade, renda e ano de ingresso na universidade.

A autoavaliação positiva de estresse foi mais prevalente em estudantes universitários que praticavam atividade física de intensidade moderada e vigorosa. Importante destacar que durante períodos de grande estresse, os estudantes podem aumentar as atividades sedentárias e diminuir as atividades físicas moderadas a vigorosas ${ }^{9}$, o que corrobora com as características dos resultados desta pesquisa, quanto a menor prevalência de estresse positivo em estudantes não praticantes de atividades físicas no lazer. De forma semelhante aos resultados observados neste estudo, adultos praticantes de atividades físicas moderadas a vigorosas de Singapura apresentaram menores chances de distress psicológico, que representa o estresse negativo, independente de indicadores sociodemográficos, como sexo e idade e, outros fatores relacionados às condições de vida e a saúde ${ }^{17}$. Além disso, os asiáticos de Singapura que apresentavam maior tempo sedentário apresentaram chances 1,3 vezes maior de avaliar o estresse como negativo ${ }^{17}$. Entretanto, no contexto brasileiro não foi observada a associação entre atividade física no lazer e autoavaliação de estresse na vida em trabalhadores da indústria ${ }^{18}$.

As possíveis explicações acerca do efeito benéfico da prática de atividades físicas no lazer sobre a autoavaliação positiva de estresse na vida baseiam-se no potencial da prática regular, de intensidade de no mínimo moderada em estimular, 
em nível cognitivo, a produção de hormônios moduladores das sensações de bem estar, assim como, a possibilidade de aumento da capacidade oxidativa do cérebro ${ }^{19}$ e desta forma minimizar a percepção subjetiva de estresse como negativo na vida. A prática sistematizada de exercícios físicos pode ser também benéfica para a proteção contra a depressão e ansiedade ${ }^{20}$, que são elementos que podem potencializar o estresse ruim.

Os resultados deste estudo mostraram maior proporção de autoavaliação positiva de estresse em homens $(35,8 \%)$, semelhante ao identificado em outros estudos com adultos ${ }^{21}$, trabalhadores da indústria ${ }^{18,22}$, assim como em estudantes universitários $^{23}$. Em pesquisa com estudantes da escola de Medicina da Arábia Saudita, as mulheres apresentaram quase três vezes mais chances (OR: 2,7; IC95\%: 1,8$3,9)$ de apresentar estresse negativo que os homens ${ }^{23}$. Além disso, vale salientar a predominância de estresse negativo para o sexo feminino ${ }^{22-24}$, possivelmente pelas exigências em relação às questões doméstica, laborais e estéticas, bem como em relação a uma maior exigência em relação à saúde, que pode contribuir com uma maior autoavaliação negativa da saúde $\mathrm{e}^{25-27}$.

Nesta pesquisa, os estudantes jovens e aqueles do primeiro ano de universidade apresentaram maior prevalência de autoavaliação de estresse positivo. No entanto, em estudantes do curso de Medicina da Arábia Saudita maiores prevalências de ausência de estresse negativo (equivalente a autoavaliação positiva de estresse) foram observados nos estudantes dos últimos anos do curso e consequentemente aqueles em idade mais avançada, especificamente, pela obtenção de maior experiência e envolvimento com as atividades da futura profissão, permitindo assim maior percepção de competência, diferentemente dos estudantes recém-ingressantes $^{23}$. Embora as diferenças entre as medidas empregadas entre os estudos, assim como os aspectos culturais entre o Brasil e Arábia Saudita e, neste estudo o levantamento de informações em diferentes cursos, possivelmente em estudantes brasileiros, a diminuição da autoavaliação do estresse positivo na vida e desta forma o aumento da avaliação negativa no final do curso possa ter relação com as exigências do período final de conclusão em virtude de trabalhos acadêmicos ${ }^{8}$, assim como a preocupação com a necessidade da inserção no mercado de trabalho, pós-conclusão do curso ${ }^{28}$.

Importante caracterizar que as limitações deste estudo são: o delineamento transversal do método, tendo em vista a impossibilidade do estabelecimento de causalidade $^{29}$, exceto para a variável sexo, o uso de uma pergunta para mensurar o estresse e assim a possibilidade de viés de resposta em função da consideração de outros fatores momentâneos sobre a autoavaliação de estresse na vida ${ }^{30}$, assim como o emprego de uma pergunta para mensurar a prática de atividades físicas no lazer em uma semana normal, que pode contribuir com o viés de memória, pela ausência de referir com precisão os tipos de atividades físicas praticadas em uma semana típica ${ }^{31}$. Além disso, o tamanho da amostra para estimar a associação entre a prática de atividades físicas no lazer de intensidade moderada representa uma limitação nas análises estatísticas.

Como pontos positivos destaca-se que o possível viés de mensuração do estresse foi minimizado pelo período curto da coleta de dados, que não coincidiu com o período de provas finais na instituição, os níveis satisfatórios de reprodutibilidade da pergunta sobre o estresse ${ }^{13}$ e o delineamento robusto deste estudo, que incluiu diferentes cursos e a amostra selecionada aleatoriamente em cada estrato. A prática de atividades físicas no lazer, de intensidade moderada a vigorosa, ou somente vigorosa representa uma importante conduta que pode ser incentivada no âmbito 
universitário por intermédio de campanhas, mas, principalmente pode ser ofertada, por meio de projetos de extensão que permitam a prática nos espaços da universidade em diferentes dias e horários. Além disso, a implementação de políticas institucionais voltadas para a ampliação do conhecimento sobre a relevância da prática de atividades físicas no lazer, juntamente com programas de promoção da prática de atividades físicas no lazer podem aumentar a proporção de praticantes e assim maximar a possibilidade de autoavaliação positiva de estresse na vida.

\section{CONCLUSÃO}

Conclui-se que a autoavaliação positiva de estresse foi prevalente entre os homens, jovens e em estudantes que estavam no primeiro ano na universidade. Os estudantes que se dedicaram a prática de atividade física no lazer apresentaram maior prevalência de estresse positivo na vida. Essa autopercepção positiva de estresse aumentou à medida que a intensidade do exercício aumentou, bem como quando foram praticadas em ambas as intensidades.

\section{Agradecimentos}

Aos estudantes universitários que participaram do inquérito de 2010 do Estudo MONISA e aos professores que contribuíram para a aplicação do instrumento de coleta de dados.

\section{Colaboradores}

Glauciambérguia de A. Pirajá idealizou a proposta de tema deste artigo, que faz parte do trabalho de conclusão de curso em nível de especialização, e realizou a redação do manuscrito. Markus V. Nahas contribuiu para a concepção do Estudo MONISA e contribuiu na revisão crítica do artigo. Aline R. Barbosa auxiliou na redação e revisão crítica do artigo. Silvio A Fonseca contribuiu na proposta metodológica do Estudo MONISA e auxiliou na revisão crítica do artigo. Thiago F. de Sousa orientou a autora do artigo, realizou as análises estatísticas do artigo, desenvolveu os procedimentos metodológicos e coordena o Estudo MONISA.

\section{REFERÊNCIAS}

1. Lipp MEN (Org.). O stress está dentro de você. São Paulo (SP): Contexto; 2000.

2. Nahas MV. Conceitos e sugestões para um estilo de vida ativo. Londrina (PR): Midiograf; 2006.

3. Goulart EJ, Lipp MEN. Estresse entre professoras do ensino fundamental de escolas públicas estaduais. Psicol Estud 2008;13(4):847-857.

4. Brandão MRF, MatsudoVKR. Stress, emoção e exercício. Rev Bras Ciên Mov 1990;4(4):95-9.

5. Yusuf S, Hawken S, Ounpuu S, et al. Effect of potentially modifiable risk factors associated with myocardial infarction in 52 countries (the INTERHEART study): case-control study. Lancet 2004;364:937-52.

6. Brandão MP, Pimentel FL, Cardoso MF. Impact of academic exposure on health status of university students. Rev Saúde Pública 2011;45(1):49-58.

7. Fontes ACD, Vianna RPT. Prevalência e fatores associados ao baixo nível de atividade física entre estudantes universitários de uma universidade pública da região Nordeste - Brasil. Rev Bras Epidemiol 2009;12(1):20-9.

8. Tyrer P. The behavior of academics under stress: A commentary on Blashfield and Reynolds. J Pers Disord 2012;26(6):835-837.

9. Cruz SY, Fabián C, Pagán I, et al. Physical activity and its associations with sociodemographic characteristics, dietary patterns, and perceived academic stress in students attending college in Puerto Rico. P R Health Sci J 2013;1:44-50. 
10. Pucci GCMF, Rech CR, Fermino RC, et al. Associação entre atividade física e qualidade de vida em adultos. Rev Saúde Pública 2012;46(1):166-79.

11. Sousa TF, Fonseca SA, Mororó José HP, et al. Estudo MONISA: características e aspectos metodológicos. Rev Bras Epidemiol 2012;15(4):924-7.

12. Luiz RR, Magnanini MF. A lógica da determinação do tamanho da amostra em investigações epidemiológicas. Cad Saúde Coletiva 2000;8(2):9-28.

13. Sousa TF, Fonseca SA, Mororó JHP, et al. Validade e reprodutibilidade do questionário Indicadores de Saúde e Qualidade de Vida de Acadêmicos (Isaq-A). Arq Ciênc Esporte 2013;1(1):21-30.

14. Simão CB, Nahas MV, Oliveira ESA.Atividade física habitual, hábitos alimentares e prevalência de sobrepeso e obesidade em universitários da Universidade do Planalto Catarinense UNIPLAC, Lages. SC. Rev Bras Ativ Fís Saúde 2006;11(1):3-12.

15. Mielke GI, Ramis TR, Campos E, et al. Atividade física e fatores associados em universitários do primeiro ano da Universidade Federal de Pelotas. Rev Bras Ativ Fís Saúde 2010;15(1):57-64.

16. Ainsworth BE, Haskell WL, Whitt MC, et al. Compendium of physical activities: an update of activity codes and MET intensities. Med Sci Sports Exerc 2000;32(9):S498-S516.

17. Sloan RA, Sawada SS, Girdano D, et al. Associations of sedentary behavior and physical activity with psychological distress: a cross-sectional study from Singapore. BMC Public Health 2013;13:885.

18. Farah BQ Barros MVG, Farias Júnior JC, et al. Percepção de estresse: associação com a prática de atividades físicas no lazer e comportamentos sedentários em trabalhadores da indústria. Rev Bras Educ Fís Esporte 2013;27(2):225-34.

19. Antunes HKM, Santos RF, Cassilhas R, et al. Exercício físico e função cognitiva: uma revisão. Rev Bras Med Esporte 2006;12(2):108-114.

20. Cheik NC, Reis IT, Heredia RAG, et al. Efeitos do exercício físico e da atividade física na depressão e ansiedade em indivíduos idosos. Rev Bras Ciên Mov 2003;11(3):45-52.

21. Sparrenberger F, Santos I, Lima RC. Associação de eventos de vida produtores de estresse e mal-estar psicológico: um estudo de base populacional. Cad Saúde Pública 2004;20(1):249-58.

22. Barros MVG, Nahas MV. Comportamentos de risco, auto-avaliação do nível de saúde e percepção de estresse entre trabalhadores da indústria. Rev Saúde Pública 2001;35(6):554-63.

23. Abdulghani HM, Alkanhal AA, Mahmoud ES, et al. Stress and its effects on medical students: A cross-sectional study at a college of medicine in Saudi Arabia.J Health Popul Nutr 2011;29(5):516-52.

24. Calais SL, Andrade LMB, Lipp MEN. Diferenças de sexo e escolaridade na manifestação de stress em adultos jovens. Psicol Refl Crít 2003;16(2):257-263.

25. Fonseca SA, Blank VLG, Barros MVG, et al. Percepção de saúde e fatores associados em industriários de Santa Catarina, Brasil. Cad Saúde Pública 2008;24(3):567-76.

26. Sousa TF, Silva KS, Garcia LMT, et al. Autoavaliação de saúde e fatores associados em adolescentes do Estado de Santa Catarina, Brasil. Rev Paul Pediatr 2010;28(4):333-9.

27. Abolfotouh MA, Bassiouni SA, Mounir GM, et al. Health-related lifestyle and risk behaviours among students living in Alexandria University hostels. EMHJ 2007;13(2):376-91.

28. Gondim SMG. Perfil profissional e mercado de trabalho: relação com a formação acadêmica pela perspectiva de estudantes universitários. Estud Psicol (Natal) 2002;7(2):299-309.

29. Bastos JLD, Duquia RP. Um dos delineamentos mais empregados em epidemiologia: estudo transversal. Sci Med 2007;17(4):229-32.

30. Sparrenberger F, Santos I, Lima RC. Epidemiologia do distress psicológico: estudo transversal de base populacional. Rev Saúde Pública 2003;37(4):434-9.

31. Baranowski T. Validity and reliability of self-report measures of physical activity: an information-processing perspective. Res QExerc Sport 1988;59:314-27. 